oesophageal $\mathrm{pH}$ monitoring data. Eur $\mathcal{F}$ Pediatr 1990; 149: 598-602.

9 Vandenplas Y, Lepoudre R, Helven R. Dependability of esophageal $\mathrm{pH}$ monitoring data in infants on cut-off limits: the oscillatory index. $f$ Pediatr Gastroenterol Nutr (in press)

\section{Elimination of high titre HIV from fibreoptic} endoscopes

SIR, - I would like to congratulate Dr Hanson and his colleagues on their encouraging paper concerning the elimination of high titre HIV from fibreoptic endoscopes. ${ }^{1}$ It confirms that thorough cleaning and removal of all organic material is the most important step in the decontamination process and necessary for disinfectants to be fully effective. ${ }^{2}$ Unfortunately, the last sentence of Dr Hanson's excellent article, which advocates the use of machines to ensure good cleaning, may be misunderstood in three ways:

(1) That trained and experienced endoscopy staff need not be employed or existing staff can be replaced by a machine;

(2) That equipment does not need manual cleaning before being put into automated systems;

(3) That even in the absence of predisinfection manual cleaning, equipment has been decontaminated effectively because it has been put through an automated 'cleaning' and disinfection cycle.

There are at present no automated disinfection machines available which can replace manual cleaning with brushes before disinfection. ${ }^{2}$ Trained and experienced staff are required ${ }^{2}$ because endoscopes and their accessories need to be dismantled according to manufacturers' instructions so that all contaminated areas can be cleaned and disinfected appropriately. In inexperienced hands contaminated instruments may be left to be cleaned at a more convenient time, allowing organic material to dry in the channels. Disinfection machines, which are often claimed to be effective cleaners, cannot carry out proper disinfection unless equipment has been cleaned manually beforehand and all organic material has been removed. The presence of any organic material will prevent adequate penetration of disinfectants. ${ }^{2}$ This has been shown in a case report where an endoscope, which was later found to be contaminated with Salmonella typhimurium, was put into an automated cleaning/disinfection machine without prior manual cleaning. ${ }^{3}$ Nine days after the presumed contamination, $S$ typhimurium could still be recovered from the endoscope despite it having gone through several automated cleaning/ disinfection cycles during that time. Another efficacy failure of an automated machine has been reported from the United States, where, after adopting an automated method of cleaning endoscopes, a hospital noted a dramatic increase in the prevalence of bile cultures positive for Pseudomonas aeruginosa. ${ }^{+}$They traced the organism to a single endoscope contaminated with $P$ aeruginosa. Although the instrument had been cleaned and disinfected repeatedly with an automated system, several channels were not adequately cleaned or dried by the washer/ disinfector.

Despite rejecting the use of automated cleaning/disinfection machines without prior manual cleaning I do advocate their appropriate use for consistent disinfection. Moreover, they reduce staff contact exposure to glutaraldehyde liquid and to vapour during the disinfection cycles, though they may increase vapour exposure during the filling and emptying of machines
(J R Babb, personal communication). Automated machines are complementary to manual cleaning and to trained and experienced staff, but cannot replace them.

CHRISTIANE S NEUMANN Gastroenterology Unit, Dudley Road Hospital, Birmingham B187QH

1 Hanson PJV, Gor D, Jeffries DJ, Collins JV. Elimination of high titre HIV from fibreoptic endoscopes. Gut 1990; 31: 657-9.

2 British Society of Gastroenterology. Cleaning and disinfection of equipment for gastrointestinal disinfection of equipment for gastrointestinal of a Working Party. Gut 1988; 29: 1134-51.

3 Hawkey PM, Davies AJ, Viant AC, et al. Contamination of endoscopes by Salmonella species. f Hosp Infect 1981; 2: 373-6.

4 Allen JI, O'Connor-Allen M, Olsen MM et al. Pseudomonas infection of the biliary system resulting from use of a contaminated endoscope. Gastroenterology 1987; 92: 759-63.

\section{Reply}

SIR, - We agree entirely with the points raised by Ms Neumann. It is only through misinterpretation of the last sentence of our paper that our views differ. Our intended emphasis was on the first part of the sentence that the large reductions in HIV achieved in our study were the result of manual cleaning. It has been suggested that endoscopes contaminated with

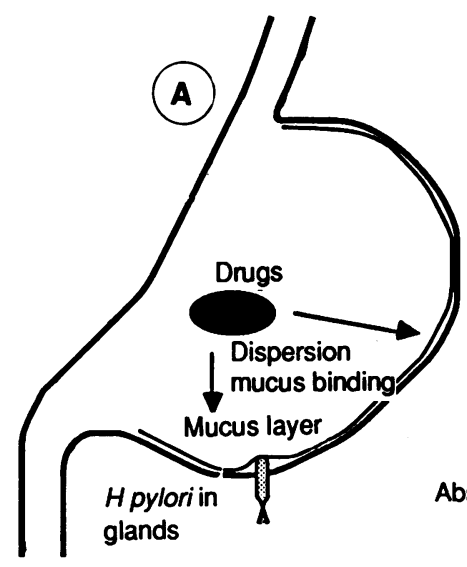

Accepted mechanism of drug delivery
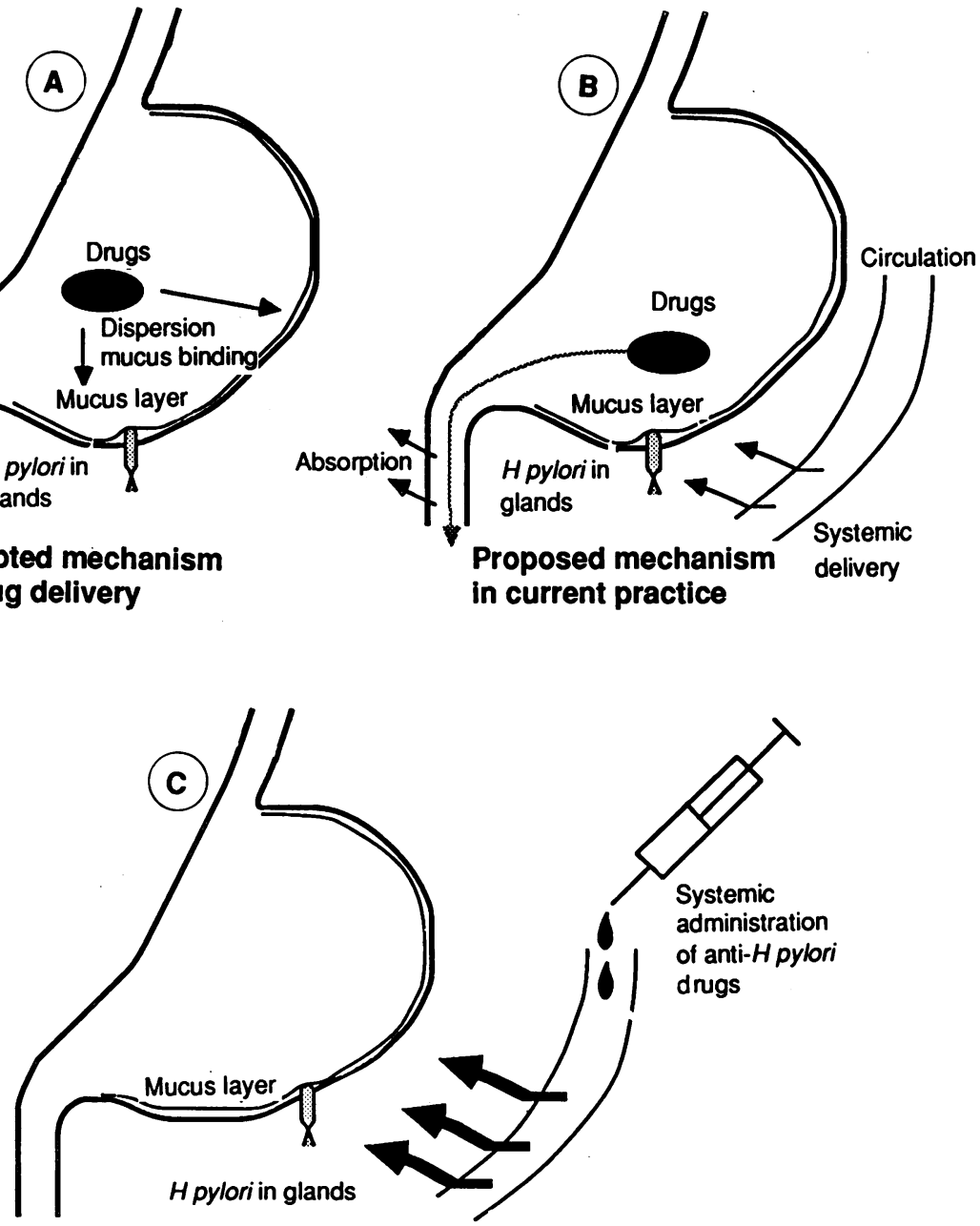

Proposed extension of current practice

Illustration of alternative concepts of delivery of xenobiotics to gastric Helicobacter pylori. (A) Presently accepted mechanism. (B) The alternative interpretation. (C) The rationale of intravenous treatment with antibiotics.
HIV must be cleaned in an automated machine and our findings do not support this view. I welcome Ms Neumann's reminder that there is no substitute for trained endoscopy personnel and that endoscopes must be precleaned before being placed in any of the currently available 'automatic' machines.

$$
\begin{aligned}
& \text { P J V HANSON } \\
& \text { Guy's Hospital, } \\
& \text { St Thomas Street, } \\
& \text { London SE I }
\end{aligned}
$$

Systemic chemotherapy for Helicobacter pylori eradication?

SIR, - Recent studies indicate the involvement of Helicobacter pylori in several upper gastrointestinal disorders including antral type B gastritis, duodenal ulcers, and non-ulcer dyspepsia. ${ }^{1-5}$ The approach to delivery of drug treatment for $H$ pylori infection is currently based on the concept of therapeutic delivery from the gastric juice after drugs have been given orally. The aim of this is eradication. Ulcer relapse rate has been found to correlate with recurrence of infection. ${ }^{6}$ Success rates range from $33-100 \%$ for immediate eradication of $H$ pylori, with many patients having a recurrence within one month of stopping treatment, ${ }^{7}$ often with the same bacteria. ${ }^{8}$ The fact that bacteria are sensitive in vitro but much less so in vivo to a number of antimicrobials has 